International Journal of Pure and Applied Mathematics

Volume 112 No. 1 2017, 115-124

ISSN: 1311-8080 (printed version); ISSN: 1314-3395 (on-line version)

url: http://www.ijpam.eu

doi: 10.12732 /ijpam.v112i1.9

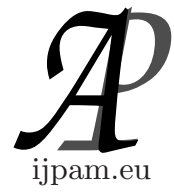

\title{
COMPLETE REDUCIBILITY OF REPRESENTATIONS OF $L$-FUZZY GROUPS
}

\author{
R. Uzbashy ${ }^{1}$, A.A. Hanano ${ }^{2}$, E. Koudsi ${ }^{3}$ \\ $1,2,3$ Department of Mathematics \\ Faculty of Science \\ Damascus University \\ Damascus, SYRIA
}

\begin{abstract}
This paper studies the complete reducibility of representations of $L$-fuzzy groups. We define the comparable family of $L$-fuzzy spaces, and prove the following theorem: Let $\widetilde{T}_{\mu}(\mathcal{V})$ be a completely reducible representation of an $L$-fuzzy group $\mu$ in a $\mu$-space $\mathcal{V}$, and $\left\{\mathcal{W}_{i}\right\}_{i \in I}$ be a family of $\mu$-subspaces of $\mathcal{V}$. If the intersection $\bigcap_{i \in I} \mathcal{W}_{i}$ is positive, then every $\mathcal{W}_{i}$ is completely reducible.

In addition, we introduce a criterion to validate the complete reducibility of $\widetilde{T}_{\mu}(\mathcal{V})$ as follows: If the representation $T: \operatorname{Supp} \mu \rightarrow G L(\operatorname{Supp} \mathcal{V})$ is completely reducible, then $\widetilde{T}_{\mu}(\mathcal{V})$ is completely reducible too. However, we prove that the inverse is not necessarily true, but it is satisfied when the set $\bigcup_{i \in I} \mathcal{W}_{i}$ is comparable.

Finally, as a result, we show that every representation of $L$-fuzzy group in any $\mu$-space $\mathcal{V}$ over the field of complex numbers is always completely reducible.
\end{abstract}

AMS Subject Classification: 20N25, 20F29

Key Words: $L$-fuzzy group, $L$-fuzzy space, $\mu$-space, completely reducible representation

\section{Introduction}

In [9], Zadeh introduced the notion of fuzzy set of a nonempty set $X$ as a function $\mu: X \rightarrow[0,1]$, and also defined the extension principles. Goguen in [1] generalized the construction of fuzzy sets by allowing the range of the extended characteristic functions to be a complete distributive lattice $L$.

Received: November 29, 2016

Revised: $\quad$ January 12, 2017

Published: January 24, 2017

(C) 2017 Academic Publications, Ltd. url: www.acadpubl.eu

${ }^{\S}$ Correspondence author 
Many researchers are engaged in extending the notions of algebra to the broader framework of the fuzzy setting. We are specifically interested in those which are related to the group theory and linear algebra. The notions of fuzzy groups and fuzzy spaces are carried out by Rosenfeld in [7], and Katsaras and Liu in [2] respectively.

The primary purpose of this paper is to study the properties of representations of $L$-fuzzy groups, especially, the complete reducibility of these representations.

\section{Preliminaries}

Throughout the paper, we assume $(L, \leq, \vee, \wedge)$ is a complete distributive lattice with an infinity $I$ and a zero $0, G$ is a finite group, $V$ is a vector space over a field $F$, and $L^{X}$ is a family of all $L$-fuzzy sets of a set $X$. If $\mu \in L^{X}$ then we write $\operatorname{Supp} \mu$ for the support set of $\mu$, where $\operatorname{Supp} \mu=\{x \in X ; \mu(x)>0\}$. When $\operatorname{Supp} \mu=X$ then $\mu$ is said to be positive, and when $\mu(x)=0$ for every $x \in X$ then $\mu$ is said to be an empty $L$-fuzzy set of $X$, and denoted by $\emptyset$.

In this section, we introduce definitions and elementary results of $L$-fuzzy groups and $L$-fuzzy spaces.

Definition 2.1 [4] Let $X, Y$ be nonempty sets. Through the extension principle of Zadeh, each function $f: X \rightarrow Y$ induces a corresponding function $\widetilde{f}: L^{X} \rightarrow L^{Y}$ defined for each $\mu \in L^{X}$ by

$$
\tilde{f}_{\mu}(y)= \begin{cases}\bigvee_{x \in f^{-1}(y)}\{\mu(x)\}, & f^{-1}(y) \neq \emptyset, \mu(x)>0 \\ 0, & \text { otherwise }\end{cases}
$$

for every $y \in Y$.

The function $\tilde{f}$ is said to be obtained from $f$ by the extension principle.

Definition 2.2[5][7] Let $(G, \cdot)$ be a finite group. An $L$-fuzzy set $\mu \in L^{G}$ is said to be an $L$-fuzzy group of $G$ if $\mu$ satisfies the properties, for every $x, y \in G$ :

1. $\mu(x \cdot y) \geq \mu(x) \wedge \mu(y)$.

2. $\mu\left(x^{-1}\right)=\mu(x)$.

Theorem 2.3 [6] If $\mu \in L^{G}$ is an L-fuzzy group, then Supp $\mu$ is a subgroup of $G$. 
Theorem 2.4 [8] Let $G, G$ be two finite groups, and $f: G \rightarrow G$ be a group homomorphism. If $\mu$ is an L-fuzzy group of $G$, then $\widetilde{f}_{\mu}$ is an L-fuzzy group of $G$, where $\tilde{f}$ is the extended function of $f$ by the extension principle.

Definition 2.5 [2][3] Let $V$ be a vector space. An $L$-fuzzy set $\mathcal{V} \in L^{V}$ is said to be an $L$-fuzzy space of $V$ if $\mathcal{V}$ satisfies the property, for every $v, u \in$ $V, \alpha, \beta \in F$ :

$$
\mathcal{V}(\alpha v+\beta u) \geq \mathcal{V}(v) \wedge \mathcal{V}(u)
$$

Definition 2.6 [3] Let $\mathcal{V} \in L^{V}$ be an $L$-fuzzy space of the vector space $V$, then $\mathcal{V}$ is said to be trivial if $\mathcal{V}=0_{\mathcal{V}(0)}$ for every $v \in V$, where $0_{\mathcal{V}(0)}$ is an $L$-fuzzy point defined as $0_{\mathcal{V}(0)} \in L^{V}$ such

$$
0_{\mathcal{V}(0)}(v)= \begin{cases}\mathcal{V}(0) & ; v=0 \\ 0 & ; \text { otherwise }\end{cases}
$$

Theorem 2.7 [3] If $\mathcal{V} \in L^{V}$ is an L-fuzzy space, then $S u p p \mathcal{V}$ is a subspace of $V$.

Definition 2.8 [3][8] Let $\mathcal{V}_{1}, \mathcal{V}_{2} \in L^{V}$ be two $L$-fuzzy spaces of $V$, then $\mathcal{V}_{1}$ is said to be included in $\mathcal{V}_{2}$, or an $L$-fuzzy subspace of $\mathcal{V}_{2}$, when $\mathcal{V}_{1}(v) \leq \mathcal{V}_{2}(v)$ for every $v \in V$, and they are said to be equal when $\mathcal{V}_{1}(v)=\mathcal{V}_{2}(v)$ for every $v \in V$.

The intersection of $\mathcal{V}_{1}$ and $\mathcal{V}_{2}$ is an $L$-fuzzy set of $V$, denoted by $\mathcal{V}_{1} \cap \mathcal{V}_{2}$, such that for every $v \in V$

$$
\left(\mathcal{V}_{1} \cap \mathcal{V}_{2}\right)(v)=\mathcal{V}_{1}(v) \wedge \mathcal{V}_{2}(v)
$$

If $\operatorname{Supp} \mathcal{V}_{1} \cap S u p p \mathcal{V}_{2}=\{0\}$, then the direct sum of $\mathcal{V}_{1}$ and $\mathcal{V}_{2}$, is defined as an $L$-fuzzy set of $V$, denoted by $\mathcal{V}_{1} \oplus \mathcal{V}_{2}$, such that for every $v \in V$

$$
\left(\mathcal{V}_{1} \oplus \mathcal{V}_{2}\right)(v)=\mathcal{V}_{1}\left(v_{1}\right) \wedge \mathcal{V}_{2}\left(v_{2}\right)
$$

where $v=v_{1}+v_{2}, v_{1} \in \operatorname{Supp} \mathcal{V}_{1}, v_{2} \in \operatorname{Supp} \mathcal{V}_{2}$.

Theorem 2.9 [3] Let $\left\{\mathcal{V}_{i}\right\}_{i \in I}$ be a family of L-fuzzy spaces of $V$, then $\bigcap_{i \in I} \mathcal{V}_{i}$ is an L-fuzzy space of $V$.

Theorem 2.10 [8] Let $\mathcal{V}_{1}, \mathcal{V}_{2} \in L^{V}$ be two L-fuzzy spaces of $V$ such that Supp $\mathcal{V}_{1} \cap S u p p \mathcal{V}_{2}=\{0\}$, then $\mathcal{V}_{1} \oplus \mathcal{V}_{2}$, is an L-fuzzy space of $V$. 
Definition 2.11 Let $\tau$ be a subset of a lattice $L$. We say that $\tau$ is comparable set in $L$ when for every pair $(\alpha, \beta) \in \tau \times \tau, \alpha$ and $\beta$ are comparable in $L$, i.e $(\alpha \wedge \beta=\alpha$ and $\alpha \vee \beta=\beta)$, or $(\alpha \wedge \beta=\beta$ and $\alpha \vee \beta=\alpha)$. The family $\left\{\mathcal{V}_{i}\right\}_{i \in I}$ of $L$-fuzzy spaces of $V$ is said to be comparable family in $L$, if the set $\bigcup_{i \in I} \operatorname{Im} \mathcal{V}_{i}$ is comparable set in $L$.

Remark 2.12 Note that if the lattice $L$ is the unit interval, $[0,1]$, then every family $\left\{\mathcal{V}_{i}\right\}_{i \in I}$ of $L$-fuzzy spaces of a vector space $V$ is a comparable family in $L$.

Theorem 2.13 Let $\left\{\mathcal{V}_{i}\right\}_{i \in I}$ be a family of an L-fuzzy spaces of a vector space $V$, and $\mathcal{V}_{1}, \mathcal{V}_{2} \in\left\{\mathcal{V}_{i}\right\}_{i \in I}$. If $\left\{\mathcal{V}_{i}\right\}_{i \in I}$ is comparable family in $L$, and $\mathcal{V}_{1}, \mathcal{V}_{2}$ satisfy $\operatorname{Supp} \mathcal{V}_{1} \cap \operatorname{Supp} \mathcal{V}_{2}=\{0\}$ then

$$
\operatorname{Supp}\left(\mathcal{V}_{1} \oplus \mathcal{V}_{2}\right)=\operatorname{Supp} \mathcal{V}_{1} \oplus \operatorname{Supp} \mathcal{V}_{2}
$$

Proof. Let $v \in \operatorname{Supp}\left(\mathcal{V}_{1} \oplus \mathcal{V}_{2}\right)$, then $\left(\mathcal{V}_{1} \oplus \mathcal{V}_{2}\right)(v)=\mathcal{V}_{1}\left(v_{1}\right) \wedge \mathcal{V}_{2}\left(v_{2}\right)$, where $v_{1} \in S u p p \mathcal{V}_{1}, v_{2} \in S u p p \mathcal{V}_{2}$, so $\mathcal{V}_{1}\left(v_{1}\right)>0, \mathcal{V}_{2}\left(v_{2}\right)>0$, that implies $v=v_{1}+v_{2} \in$ $\operatorname{Supp} \mathcal{V}_{1} \oplus \mathcal{V}_{2}$ therefore

$$
\operatorname{Supp}\left(\mathcal{V}_{1} \oplus \mathcal{V}_{2}\right) \subseteq \operatorname{Supp} \mathcal{V}_{1} \oplus \operatorname{Supp} \mathcal{V}_{2}(\mathbf{2 . 1 3 . 1})
$$

Let $v \in S u p p \mathcal{V}_{1} \oplus S u p p \mathcal{V}_{2}$, then $v$ is written as $v=v_{1}+v_{2}$ where $v_{1} \in$ $S u p p \mathcal{V}_{1}, v_{2} \in S u p p \mathcal{V}_{2}$, then $\mathcal{V}_{1}\left(v_{1}\right)>0, \mathcal{V}_{2}\left(v_{2}\right)>0$. Since $\mathcal{V}_{1}, \mathcal{V}_{2} \in\left\{\mathcal{V}_{i}\right\}_{i \in I}$ and the family $\left\{\mathcal{V}_{i}\right\}_{i \in I}$ is comparable in $L, \mathcal{V}_{1}\left(v_{1}\right) \wedge \mathcal{V}_{2}\left(v_{2}\right)>0$, so $\left(\mathcal{V}_{1} \oplus \mathcal{V}_{2}\right)(v)>0$, and $v \in \operatorname{Supp}\left(\mathcal{V}_{1} \oplus \mathcal{V}_{2}\right)$, therefore

$$
\operatorname{Supp} \mathcal{V}_{1} \oplus \operatorname{Supp} \mathcal{V}_{2} \subseteq S u p p\left(\mathcal{V}_{1} \oplus \mathcal{V}_{2}\right)(\mathbf{2 . 1 3 . 2})
$$

from $(2.13 .1),(2.13 .2)$ we have

$$
\operatorname{Supp}\left(\mathcal{V}_{1} \oplus \mathcal{V}_{2}\right)=\operatorname{Supp} \mathcal{V}_{1} \oplus \operatorname{Supp} \mathcal{V}_{2}
$$

\section{Representations of $L$-Fuzzy Groups}

Throughout the paper, we shall consider the following definitions and the results about representations of $L$-fuzzy groups which we introduced all in our work $[8]$.

Definition 3.1 Let $\mu \in L^{G}$ ba an $L$-fuzzy group, and 


$$
\begin{aligned}
T: G & \rightarrow G L(V) \\
g & \mapsto T_{g}
\end{aligned}
$$

be a linear representation of the group $G$ in the vector space $V$.

Through the extension principle, $T$ is extended to the function:

$$
\begin{aligned}
\widetilde{T}: L^{G} & \rightarrow L^{G L(V)} \\
\mu & \mapsto \widetilde{T}_{\mu}
\end{aligned}
$$

where

$$
\widetilde{T}_{\mu}(f)= \begin{cases}\bigvee_{g \in T^{-1}(f)}\{\mu(g)\} & ; T^{-1}(f) \neq \emptyset, \mu(g)>0 \\ 0 & ; \text { otherwise }\end{cases}
$$

for every $f \in G L(V)$.

We call the $L$-fuzzy group $\widetilde{T}_{\mu}$ of $G L(V)$, a representation of $L$-fuzzy group $\mu$ in $V$.

Definition 3.2 An $L$-fuzzy space $\mathcal{V} \in L^{V}$ is said to be a $\mu$-space when there exists a representation $\widetilde{T}_{\mu}$, so that

$$
\mathcal{V}(v)=\mathcal{V}\left(T_{g}(v)\right)
$$

for every $g \in S u p p \mu, v \in S u p p \mathcal{V}$

Definition 3.3 Let $\widetilde{T}_{\mu}$ be a representation of the $L$-fuzzy group $\mu$ in the vector space $V$, and $\mathcal{V}$ be a $\mu$-space. The representation of $L$-fuzzy group $\mu$ in $\mathcal{V}$ is an $L$-fuzzy group of $G L(V)$, denoted by $\widetilde{T}_{\mu}(\mathcal{V})$, which obtained by extending the representation $T$ of the finite group $S u p p \mu$ in the vector space $S u p p \mathcal{V}$, where $T(g)=\left.T_{g}\right|_{\text {Supp } \mathcal{V}}$ for every $g \in \operatorname{Supp} \mu$.

That means for every $f \in G L(S u p p \mathcal{V})$

$$
\widetilde{T}_{\mu}(\mathcal{V})(f)= \begin{cases}\bigvee_{g \in T^{-1}(f)}\{\mu(g)\} & ; T^{-1}(f) \neq \emptyset \\ 0 & ; \text { otherwise }\end{cases}
$$

Theorem 3.4 Let $\mathcal{V}$ be a $\mu$-space, then $S u p p \mathcal{V}$ is a Supp $\mu$-space, i.e for every $v \in \operatorname{Supp} \mathcal{V}$ and $g \in \operatorname{Supp} \mu, T_{g}(v) \in \operatorname{Supp} \mathcal{V}$.

Theorem 3.5 If $\left\{\mathcal{V}_{i}\right\}_{i \in I}$ is any family of $\mu$-spaces of $V$, then $\bigcap_{i \in I} \mathcal{V}_{i}$ is a $\mu$-space of $V$.

Definition 3.6 Let $\widetilde{T}_{\mu}(\mathcal{V})$ be a representation of the $L$-fuzzy group $\mu$ in an $L$-fuzzy space $\mathcal{V}$, then $\widetilde{T}_{\mu}(\mathcal{V})$ is said to be irreducible if the $\mu$-space $\mathcal{V}$ has no 
non-trivial $\mu$-space $\mathcal{W}$ where $\mathcal{W} \subset \mathcal{V}$. Otherwise the representation $\widetilde{T}_{\mu}(\mathcal{V})$ is reducible.

Definition 3.7 Let $\widetilde{T}_{\mu}(\mathcal{V})$ be a representation of the $L$-fuzzy group $\mu$ in an $L$-fuzzy space $\mathcal{V}$, then $\widetilde{T}_{\mu}(\mathcal{V})$ is said to be completely reducible, or equivalently $\mathcal{V}$ is said to be completely reducible, if there exists for every $\mu$-space $\mathcal{W}$ where $\mathcal{W} \subset \mathcal{V}$, a $\mu$-space $\mathcal{U}$ where $\mathcal{U} \subset \mathcal{V}$, so that $\mathcal{W} \oplus \mathcal{U} \subseteq \mathcal{V}$.

Remark 3.8 It is easy to verify that every irreducible representation $\widetilde{T}_{\mu}(\mathcal{V})$ is completely reducible representation.

Throughout this paper, we shall consider the previous difintion of the representation of $L$-fuzzy groups in $\mu$-spaces.

\section{Completely Reducibile Representations of $L$-Fuzzy Groups}

In this section, we present a study of the completely reducible representations of $L$-fuzzy groups, and introduce in the theorem 3.9 a criterion to validate the complete reducibility of these representations.

Theorem 4.1 Let $\widetilde{T}_{\mu}(\mathcal{V})$ be a completely reducible representation of an $L$ fuzzy group $\mu$ in a $\mu$-space $\mathcal{V}$, and $\left\{\mathcal{W}_{i}\right\}_{i \in I}$ be a family of $\mu$-subspaces of $\mathcal{V}$. If the intersection $\bigcap_{i \in I} \mathcal{W}_{i}$ is positive, then every $\mu$-space $\mathcal{W}_{i}$ is completely reducible.

Proof. Let $\widetilde{T}_{\mu}(\mathcal{V})$ be a completely reducible representation, and $\mathcal{W}_{i}$ be a $\mu$-subspace from the family $\left\{\mathcal{W}_{i}\right\}_{i \in I}$. We distinguish two different cases:

(i) There is no $\mu$-subspace of $\mathcal{V}$ in the family $\left\{\mathcal{W}_{i}\right\}_{i \in I}$ included in $\mathcal{W}_{i}$, then $\mathcal{W}_{i}$ is irreducible, hence it is completely reducible.

(ii) Let $\mathcal{W}_{j} \in\left\{\mathcal{W}_{i}\right\}_{i \in I}$ so that $\mathcal{W}_{j} \subset \mathcal{W}_{i}$, and we want to prove that there is a $\mu$-subspace $\mathcal{W}_{k} \in\left\{\mathcal{W}_{i}\right\}_{i \in I}$ so that $\mathcal{W}_{k} \subset \mathcal{W}_{i}$ and the inclusion $\mathcal{W}_{k} \oplus \mathcal{W}_{j} \subseteq \mathcal{W}_{i}$ is satisfied.

Since $\widetilde{T}_{\mu}(\mathcal{V})$ is completely reducible, and $\mathcal{W}_{j} \subset \mathcal{W}_{i} \subset \mathcal{V}$, there is a $\mu$ subspace $X \in\left\{\mathcal{W}_{i}\right\}_{i \in I}$ of $\mathcal{V}$ so that

$$
\mathcal{W}_{i} \oplus X \subseteq \mathcal{V}(4.1 .1)
$$

By the theorem 3.5 and since $\bigcap_{i \in I} \mathcal{W}_{i}$ is positive, $\mathcal{W}_{i} \cap X \in L^{V}$ is a $\mu$-space, and satisfies $\mathcal{W}_{i} \cap X \neq \emptyset$.

In addition, for every $v \in V$

$$
\left(\mathcal{W}_{j} \oplus\left(\mathcal{W}_{i} \cap X\right)\right)(v)=\mathcal{W}_{j}\left(v_{1}\right) \wedge\left(\mathcal{W}_{i} \cap X\right)\left(v_{2}\right)
$$


where $v_{1} \in \operatorname{Supp} \mathcal{W}_{j}, v_{2} \in \operatorname{Supp}\left(\mathcal{W}_{i} \cap X\right)$. Hence

$$
\left(\mathcal{W}_{j} \oplus\left(\mathcal{W}_{i} \cap X\right)\right)(v)=\mathcal{W}_{j}\left(v_{1}\right) \wedge\left(\mathcal{W}_{i}\left(v_{2}\right) \wedge X\left(v_{2}\right)\right)
$$

Since the operation $\wedge$ is associative, and $\mathcal{W}_{j} \subset \mathcal{W}_{i}$

$$
\begin{aligned}
\left(\mathcal{W}_{j} \oplus\left(\mathcal{W}_{i} \cap X\right)\right)(v) & =\left(\mathcal{W}_{j}\left(v_{1}\right) \wedge \mathcal{W}_{i}\left(v_{2}\right)\right) \wedge X\left(v_{2}\right) \\
& \leq\left(\mathcal{W}_{i}\left(v_{1}\right) \wedge \mathcal{W}_{i}\left(v_{2}\right)\right) \wedge X\left(v_{2}\right) \\
& \leq \mathcal{W}_{i}\left(v_{1}+v_{2}\right) \wedge X\left(v_{2}\right) \\
& =\mathcal{W}_{i}(v) \wedge X\left(v_{2}\right)
\end{aligned}
$$

We know, through the lattice theory, that for every $\alpha, \beta \in L$, then $\alpha \wedge \beta \leq$ $\alpha, \alpha \wedge \beta \leq \beta$, hence for every $v \in V$ we have

$$
\left(\mathcal{W}_{j} \oplus\left(\mathcal{W}_{i} \cap X\right)\right)(v) \leq \mathcal{W}_{i}(v)
$$

On the other hand,

$$
\operatorname{Supp} \mathcal{W}_{i} \cap \operatorname{Supp} \mathcal{W}_{j}=\operatorname{Supp}\left(\mathcal{W}_{i} \cap \mathcal{W}_{j}\right)
$$

for any $\mu$-subspaces $\mathcal{W}_{i}, \mathcal{W}_{j} \in\left\{\mathcal{W}_{i}\right\}_{i \in I}$, because

1. for every $v \in \operatorname{Supp}\left(\mathcal{W}_{i} \cap \mathcal{W}_{j}\right)$ then $\mathcal{W}_{i}(v) \wedge \mathcal{W}_{j}(v)>0$ hence $\mathcal{W}_{i}(v)>0$ and $\mathcal{W}_{j}(v)>0$ therefore $v \in \operatorname{Supp} \mathcal{W}_{i} \cap \operatorname{Supp} \mathcal{W}_{j}$, hence $\operatorname{Supp}\left(\mathcal{W}_{i} \cap \mathcal{W}_{j}\right) \subseteq$ $\operatorname{Supp} \mathcal{W}_{i} \cap \operatorname{Supp} \mathcal{W}_{j}$.

2. Since the intersection of any $\mu$-subspaces is positive, for every $v \in S u p p \mathcal{W}_{i} \cap$ $\operatorname{Supp} \mathcal{W}_{j}$ means $\mathcal{W}_{i}(v)>0$ and $\mathcal{W}_{j}>0$, leads $v \in \operatorname{Supp}\left(\mathcal{W}_{i} \cap \mathcal{W}_{j}\right)$, hence $\operatorname{Supp} \mathcal{W}_{i} \cap \operatorname{Supp} \mathcal{W}_{j} \subseteq \operatorname{Supp}\left(\mathcal{W}_{i} \cap \mathcal{W}_{j}\right)$.

From (4.1.1),(4.1.2), we have $\operatorname{Supp} \mathcal{W}_{i} \cap \operatorname{Supp} X=\{0\}$, and then $\operatorname{Supp}\left(\mathcal{W}_{i} \cap\right.$ $X)=\{0\}$, that brings the fact $\operatorname{Supp} \mathcal{W}_{j} \cap \operatorname{Supp}\left(\mathcal{W}_{i} \cap X\right)=\{0\}$.

So we conclued that the $\mu$-subspace $\mathcal{W}_{k}$ we look for is the intersection $\mathcal{W}_{i} \cap X$, and the $\mu$-subspace $\mathcal{W}_{i}$ is completely reducible.

Theorem 4.2 Let $\widetilde{T}_{\mu}(\mathcal{V})$ be a representation of an L-fuzzy group $\mu$ in a $\mu$-space $\mathcal{V}$. If the representation $T: S u p p \mu \rightarrow G L(S u p p \mathcal{V})$ is completely reducible, then $\widetilde{T}_{\mu}(\mathcal{V})$ is completely reducible too.

Proof. (i) If the representation $\widetilde{T}_{\mu}(\mathcal{V})$ is irreducible, then by the remark 3.8 it is completely reducible.

(ii) Let $\widetilde{T}_{\mu}(\mathcal{V})$ be a reducible representation, then there exists a $\mu$-space $\mathcal{W} \in L^{V}$ such $\emptyset \neq \mathcal{W} \subset \mathcal{V}$. By the theorem $3.4 S u p p \mathcal{W}$ is $S u p p \mu$-space and included in the vector space $\operatorname{Supp} \mathcal{V}$, and since the representation $T$ is completely reducible, there is a $S u p p \mu$-space, $\mathcal{U}$, such 


$$
\operatorname{Supp} \mathcal{V}=\operatorname{Supp} \mathcal{W} \oplus \mathcal{U}(\mathbf{4 . 2 . 1})
$$

Let $\widetilde{\mathcal{U}} \in L^{S u p p \mathcal{V}}$ where

$$
\widetilde{\mathcal{U}}(v)= \begin{cases}\mathcal{V}(v) & ; v \in \mathcal{U} \\ 0 & ; \text { otherwise }\end{cases}
$$

for every $v \in S u p p \mathcal{V}$.

The $L$-fuzzy set $\widetilde{\mathcal{U}}$ is a subspace of the vectoe space $S u p p \mathcal{V}$ because for every $\alpha, \beta \in F$ and $v_{1}, v_{2} \in S u p p \mathcal{V}$

$$
\begin{aligned}
\widetilde{\mathcal{U}}\left(\alpha v_{1}+\beta v_{2}\right) & = \begin{cases}\mathcal{V}\left(\alpha v_{1}+\beta v_{2}\right) & ; v_{1}, v_{2} \in \mathcal{U} \\
0 & ; \text { otherwise }\end{cases} \\
& \leq \begin{cases}\mathcal{V}\left(v_{1}\right) \wedge \mathcal{V}\left(v_{2}\right) & ; v_{1}, v_{2} \in \mathcal{U} \\
0 & ; \text { otherwise }\end{cases} \\
& =\tilde{\mathcal{U}}\left(v_{1}\right) \wedge \tilde{\mathcal{U}}\left(v_{2}\right)
\end{aligned}
$$

Since $\mathcal{V}$ is a $\mu$-space, for every $u \in S u p p \widetilde{\mathcal{U}}$ and $g \in S u p p \mu$

$\widetilde{\mathcal{U}}(u)=\mathcal{V}(u)=\mathcal{V}\left(T_{g}(u)\right)=\widetilde{\mathcal{U}}\left(\left(T_{g}\right)(u)\right)$. Therefore the $L$-fuzzy set $\widetilde{\mathcal{U}}$ is a $\mu$-space.

On the other hand, from (4.2.1) we have $\operatorname{Supp} \mathcal{W} \cap \mathcal{U}=\{0\}$, and for every $v \in \operatorname{Supp} \mathcal{V}$

$$
(\mathcal{W} \oplus \widetilde{\mathcal{U}})(v)=\mathcal{W}(w) \wedge \widetilde{\mathcal{U}}(u)
$$

where $w \in S u p p \mathcal{W}, u \in S u p p \widetilde{\mathcal{U}}$ then

$$
\begin{aligned}
(\mathcal{W} \oplus \widetilde{\mathcal{U}})(v) & =\mathcal{W}(w) \cap \mathcal{V}(u) \\
& \leq \mathcal{V}(w) \cap \mathcal{V}(u) \\
& \leq \mathcal{V}(w+u) \\
& =\mathcal{V}(v)
\end{aligned}
$$

Hence $\mathcal{W} \oplus \widetilde{\mathcal{U}} \subseteq \mathcal{V}$. We show that for every non-trivial $\mu$-space $\mathcal{W} \subset \mathcal{V}$ there exists a $\mu$-space $\widetilde{\mathcal{U}}$ so that $\mathcal{W} \oplus \widetilde{\mathcal{U}}$. Which means $\widetilde{T}_{\mu}(\mathcal{V})$ is completely reducible representation.

Notice 4.3 The inverse of the previous criterion is not necessarily true, but it is satisfied when the set $\bigcup_{i \in I} \mathcal{W}_{i}$ is comparable, where $\left\{\mathcal{W}_{i}\right\}_{i \in I}$ is a family of $\mu$-subspaces of $\mathcal{V}$. 
Proof. Let $\widetilde{T}_{\mu}(\mathcal{V})$ be a completely reducible representation, and $H$ be a Supp $\mu$-space such $\emptyset \neq H \subset S u p p \mathcal{V}$.

Let $\widetilde{H} \in L^{\operatorname{Supp} \mathcal{V}}$ where

$$
\widetilde{H}(v)= \begin{cases}\mathcal{V}(v) & ; v \in H \\ 0 & ; \text { otherwise }\end{cases}
$$

By $3.4, \widetilde{H}$ is a $\operatorname{Supp} \mu$-space, also we have $\operatorname{Supp} \widetilde{H}=H$.

Since the representation $\widetilde{T}_{\mu}(\mathcal{V})$ is completely reducible, there exsists a $\mu$ space $\mathcal{W} \in L^{\operatorname{Supp} \mathcal{V}}$ such $\mathcal{W} \oplus \widetilde{H} \subseteq \mathcal{V}$ and $\operatorname{Supp} \mathcal{W} \cap H=\{0\}$. So $\operatorname{Supp}(\mathcal{W} \oplus \widetilde{H}) \subseteq$ Supp $\mathcal{V}$, and by (2.13.1)

$$
\operatorname{Supp}(\mathcal{W} \oplus \widetilde{H}) \subseteq \operatorname{Supp} \mathcal{W} \oplus \operatorname{Supp} \widetilde{H} \text { (4.3.1) }
$$

Since $\mathcal{W}, \widetilde{H}$ are two $L$-fuzzy spaces included in $\mathcal{V}$ then

$$
\text { Supp } \mathcal{W} \oplus \operatorname{Supp} \widetilde{H} \subseteq S u p p \mathcal{V}
$$

However, the inverse inclusion in (4.3.2) is not necessarily satisfied otherwise the set $\bigcup_{i \in I} \mathcal{W}_{i}$ is comparable, by $(2.13 .2)$.

Then we have

$$
\operatorname{Supp}(\mathcal{W} \oplus \widetilde{H})=\operatorname{Supp} \mathcal{W} \oplus \operatorname{Supp} \widetilde{H}
$$

equivalently,

$$
\operatorname{Supp}(\mathcal{W} \oplus \widetilde{H})=\operatorname{Supp} \mathcal{W} \oplus H
$$

so $\operatorname{Supp} \mathcal{V}=\operatorname{Supp} \mathcal{W} \oplus H$ holds. That confirms our assumption that the representation $T$ is completely reducible.

Result 4.4 Every representation $\widetilde{T}_{\mu}(\mathcal{V})$, where $\mathcal{V}$ is over the field of complex numbers, is completely reducible.

Proof. (i) If the representation $\widetilde{T}_{\mu}(\mathcal{V})$ is irreducible, then it is completely reducible.

(ii) Let $\mathcal{H}$ be a non-trivial $\mu$-space such $\mathcal{H} \subset \mathcal{V}$, by theorem $3.4 S u p p \mathcal{H}$ is a Supp $\mu$-space. Since the field we represent over is the field of complex numbers, then the representation $T: \rightarrow G L(S u p p \mathcal{V})$ is completely reducible, and then

$$
\operatorname{Supp} \mathcal{V}=\operatorname{Supp} \mathcal{H} \oplus(\operatorname{Supp} \mathcal{H})^{\perp}
$$

where

$$
(\operatorname{Supp} \mathcal{H})^{\perp}=\{v \in \operatorname{Supp} \mathcal{V}:\langle v, h\rangle=0 ; \forall h \in S u p p \mathcal{H}\} .
$$

Let $\mathcal{J} \in L^{S u p p \mathcal{V}}$ be an $L$-fuzzy set of $S u p p \mathcal{V}$ where 


$$
\mathcal{J}(v)= \begin{cases}\mathcal{V}(v) & ; v \in(\text { Supp } \mathcal{H})^{\perp} \\ 0 & ; \text { otherwise }\end{cases}
$$

Likewise we showed in the proof of the previous theorem, the $L$-fuzzy set $\mathcal{J}$ is a $\mu$-space such $\mathcal{H} \oplus \mathcal{J} \subseteq \mathcal{V}$, hence the representation $\widetilde{T}_{\mu}(\mathcal{V})$ where $\mathcal{V}$ is over the field of complex numbers, is completely reducible.

\section{References}

[1] J. A. Goguen, submitted by L. Zadeh, L-fuzzy Sets, Journal of Mathematical Analysis and Applications, 18, No. 1 (1967), 145-174, doi: 10.1016/0022-247X(67)90189-8.

[2] D. B. Liu, A. K. Katsaras, Fuzzy vector spaces and fuzzy topological vector spaces, Journal of Mathematics Analysis and Applications, 58, No. 1 (1977), 135-146, doi: 10.1016/0022-247X(77)90233-5.

[3] P. Lubczonok, Fuzzy vector spaces, Fuzzy Sets and System, 38, No. 3 (1990), 329-343, doi: 10.1016/0165-0114(90)90206-L.

[4] J.N. Mordeson, P.S. Nair, Fuzzy Mathematics: An Introduction for Engineers and Scientists, 20 of Janusz Kacprzyk, 20 of Studies in fuzziness and soft computing SpringerVerlag company (2001), 89-112, doi: 10.1007/978-3-7908-1808-6.

[5] J.N. Mordeson, K.R. Bhutani, A. Rosenfeld, Fuzzy Group Theory, 182 of Studies in Fuzziness and Soft Computing, Springer Berlin Heidelberg (2005), 344-350, doi: $10.1007 / \mathrm{b} 12359$.

[6] S. Ovchinnikov, On the Image of an L-Fuzzy Group, Mathematics Department, San Francisco State University, 94, Issue 1 (1998), 129-131, doi: 10.1016/S0165-0114(96)00361-2.

[7] A. Rosenfeld, Fuzzy groups, Journal of Mathematics Analysis and Applications, 35, No. 3 (1971), 512-517, doi: 10.1016/0022-247X(71)90199-5.

[8] R. Uzbashy, A.A. Hanano, E. Koudsi, An Extension of Maschke's Theorem to the Representations of L-Fuzzy Groups, Mathematics Department, Damascus University, Damascus, Syria (2015), In Press.

[9] L.A. Zadeh, Fuzzy sets, Information and Control, 8, No. 3 (1965), 338-353, doi: 10.1016/S0019-9958(65)90241-X. 